\title{
Infodemic during an epidemic
}

"We're not just fighting an epidemic; we're fighting an infodemic," were the words of Tedros Adhanom Ghebreyesus, director-general of the World Health Organization (WHO), referring to the continually circulating false news about the Covid-19 pandemic crisis. WHO explains that infodemics are an excessive amount of both real and inaccurate information about a problem, making it challenging to identify a solution, they create confusion in the population by spreading erroneous information, misinforming with apparently real data. The amount of time and other essential resources that can be affected by this problem make it a priority to combat ${ }^{1}$.

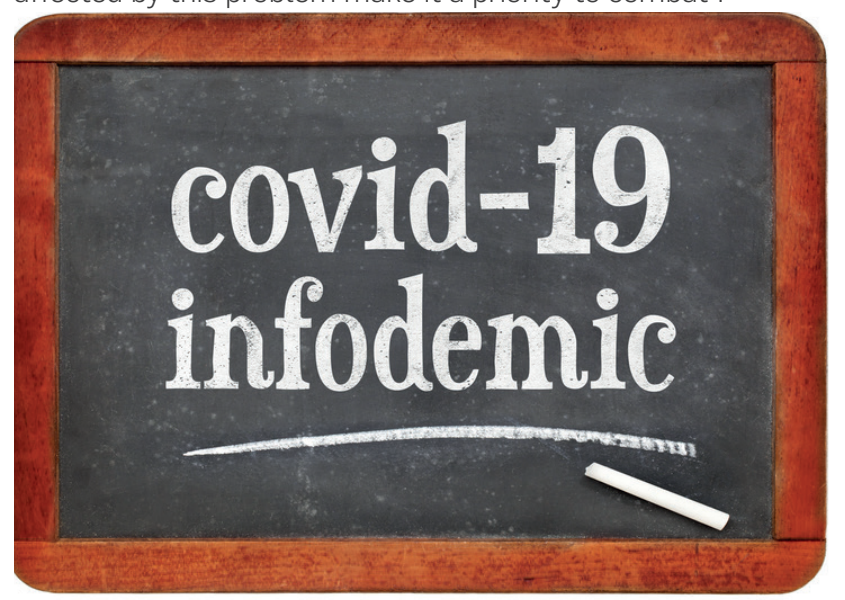

Figura 1. Covid-19 infodemic - coronavirus pandemic information overload concept

The existence of social networks and the internet is for better or for worse is one of the differentiating factors between this pandemic and the previous ones. From the manipulation of information for ideological purposes or material gain to simple jokes in the form of memes or chains on social networks, this disinformation can have substantial repercussions on the population by generating panic ${ }^{2}$. The clickbait nourishes many of these publications to its rapid spread reaching large sectors of the population in a matter of hours. This erroneous information presented by some media or shared on social networks has covered everything related to the virus from where it was generated to how it is spread. For this reason, pages like the World Health Organization have devoted themselves to destroying myths and correcting erroneous information such as those that claim that sunlight, warm weather, and drinking water can affect the coronavirus ${ }^{3}$.

An excellent way to avoid this problem and get reliable information is according to Neil Walsh, chief of the Cybercrime and Anti-Money Laundering Section of the United Nations Office on Drugs and Crime (UNODC), is to visit the pages of official sources and instructed on the subject as the World Health Organization (WHO) and the United Nations ${ }^{1}$.
To combat disinformation, UNESCO provided in its Media and Information Literacy Curriculum for teachers, a series of actions to consider when consuming information ${ }^{4}$.

- Verify the veracity of the sources in cases when the information comes from non-formal news media.

- Search for sources within any information we review.

- Know which are the main channels and providers of truthful information.

- Verify the information with other reputable media sources.

- Do not share or promote information without first checking its sources.

It is important to note that the vast majority of fake news generators can make it credible by including fake quotes, images, figures, data, and including the names of public personalities related to the topic ${ }^{3}$. Critical thinking and analysis are of utmost importance in the face of this problem. The need to compare sources and go to official sources of information is essential to avoid further damage.

Bionatura journal recommends the following information sources that have been proven truthful during the current health crisis in addition to those already mentioned before:

Open-Access Data and Computational Resources to Address COVID-19: https://datascience.nih.gov/covid-19open-access-resources

Centers for Disease Control and Prevention: https://www. cdc.gov/coronavirus/2019-nCoV/lab/index.html

Covid19.21wecan.com https://covid19.21wecan.com/ covid19en/index.shtml

\section{Bibliographic references}

1. UN. UN tackles' infodemic' of misinformation and cybercrime in COVID-19 crisis I United Nations. https://www.un.org/en/un-coronavirus-communications-team/un-tackling-'infodemic'-misinformation-and-cybercrime-covid-19 (2020).

2. Robson, D. Why smart people believe coronavirus myths. BBC (2020).

3. Naughton, J. Fake news about Covid-19 can be as dangerous as the virus. The Guardian (2020).

4. Tips to avoid online misinformation during the coronavirus crisis. https://www.bbva.com/en/tips-to-avoid-online-misinformation-during-the-coronavirus-crisis/. 